Highly Energetic Physical Processes and

Mechanisms for Emission from Astrophysical Plasmas

IAU Symposium, Vol. 195, 2000

P. C. H. Martens, S. Tsuruta, and M. A. Weber, eds.

\title{
Which Forces Accelerate Jets?
}

\author{
S. X. Kato \\ Department of Astronomy, University of Tokyo, Japan
}

T. Kudoh

National Astronomical Observatory, Japan

K. Shibata

Kwasan Observatory, Kyoto University, Japan

\begin{abstract}
We performed 2.5-dimensional, nonsteady MHD simulations of jets from geometrically thin accretion disks and investigated the acceleration forces of jets in detail.
\end{abstract}

Astrophysical jets are observed in many objects: active galactic nuclei, young stellar objects, X-ray binary systems, and so on, but we do not know their formation and acceleration mechanism well. By extending the MHD wind/jet model (e.g., Uchida \& Shibata 1985; Shibata \& Uchida 1986; Kudoh, Matsumoto, \& Shibata 1995; Matsumoto et al. 1996) which is the most promising model for jet formation and acceleration, we performed 2.5D, axisymmetric, nonsteady MHD simulations of jets from geometrically thin disks, including the dynamics of the disk. In our simulations, we assumed (1) adiabatic conditions $(\gamma=5 / 3)$, (2) that the self-gravity of the disk can be neglected, (3) that the hot corona keeps hydrostatic equilibrium, (4) that the dynamics are nonrelativistic, and (5) that the gravity is given by the gravitational potential produced by a point mass at the origin. In this model, the initial disk is assumed to be in dynamical equilibrium (e.g., see Abramowicz, Jaroszynski, \& Sikora 1978). Two important, nondimensional parameters are $E_{\mathrm{mg}}$ and $E_{\mathrm{th}}$, defined at the densest point in the initial disk $((r, z)=(1.0,0)) . E_{\mathrm{mg}}$ is the square of the ratio of Alfvén velocity to Keplerian velocity and shows the initial magnetic field strength, while $E_{\text {th }}$ (fixed at $2.0 \times 10^{-3}$ here) is the square of the ratio of the sound velocity to Keplerian velocity and shows the temperature of the disk.

In order to investigate the acceleration force of jets, we used Lagrangian particles (test particles, see Figures 1a, b). For each of the open circles, we measured the acceleration force parallel to the stream lines. Our results are as follows:

- In the weak magnetic field case, magnetic pressure accelerates jets. $\left(E_{\mathrm{mg}}<\right.$ $5.0 \times 10^{-3}$.)

- In the strong magnetic field case, magnetocentrifugal force is effective (e.g., see Blandford \& Payne 1982). $\left(E_{\mathrm{mg}} \geq 5.0 \times 10^{-3}\right.$.) 
Even in the sub-Keplerian case (the initial velocity of the disk is less than the Keplerian velocity) and the thick disk case (e.g., Kudoh, Matsumoto, \& Shibata 1998), our results can be applied.
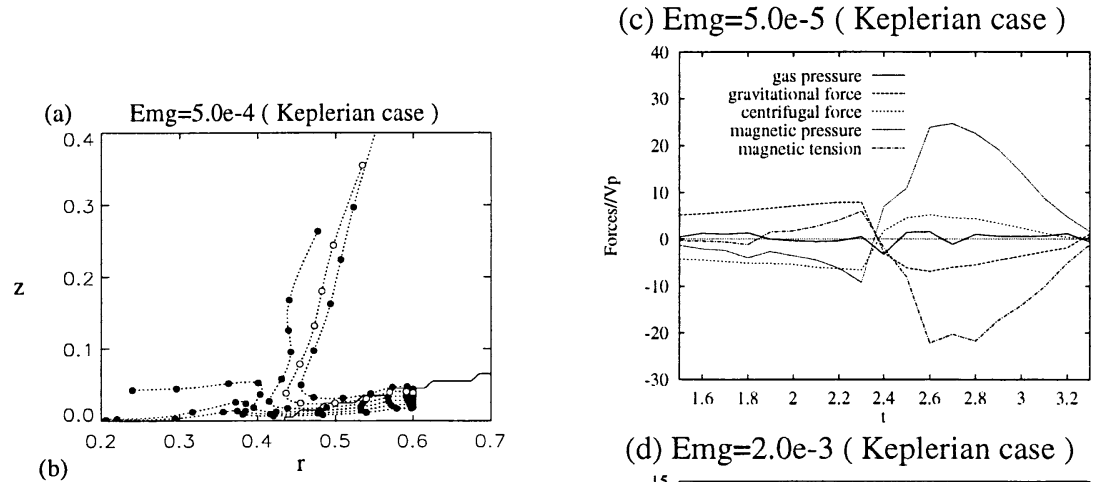

(b)
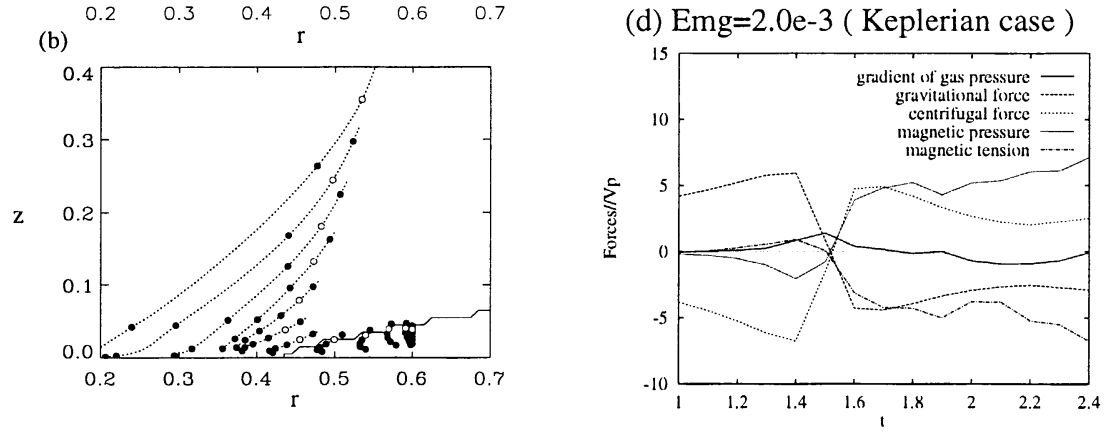

Figure 1. (a) The trajectories of Lagrangian particles. We investigated the acceleration force with the open circles. (b) The time evolution of the magnetic field located on $r=0.6$ initially. (c) The acceleration force (weak magnetic field case). Jets are ejected at about $t=2.4$ and are mainly accelerated by magnetic pressure. (d) The strong field case. Compared to (c), the magnetocentrifugal force is effective.

\section{References}

Abramowicz, M., Jaroszynski, M., \& Sikora, M. 1978, A\&A, 63, 221

Blandford, R. D., \& Payne, D. G. 1982, MNRAS, 199, 883

Kato, X. S., 1999, M.S. thesis, University of Tokyo

Kudoh, T., Matsumoto, R., \& Shibata, K. 1998, ApJ, 508, 186

Kudoh, T., \& Shibata, K. 1995, ApJ, 452, L41

Kudoh, T., \& Shibata, K. 1997, ApJ, 474, 362

Matsumoto, R., Uchida, Y., Hirose, S., Shibata, K., Hayashi, M. R., Ferrari, A., Bodo, G., \& Norman, C. 1996, ApJ, 461, 115

Shibata, K., \& Uchida, Y. 1986, PASJ, 38, 631

Uchida, Y., \& Shibata, K. 1985, PASJ, 37, 515 